\title{
Eagle Syndrome as a Cause of Cerebral Venous Sinus Thrombosis
}

\author{
Fu-Liang Zhang (D)*, Hong-Wei Zhou*, Zhen-Ni Guo, Yi Yang
}

Keywords: Eagle syndrome, Cerebral venous sinus thrombosis

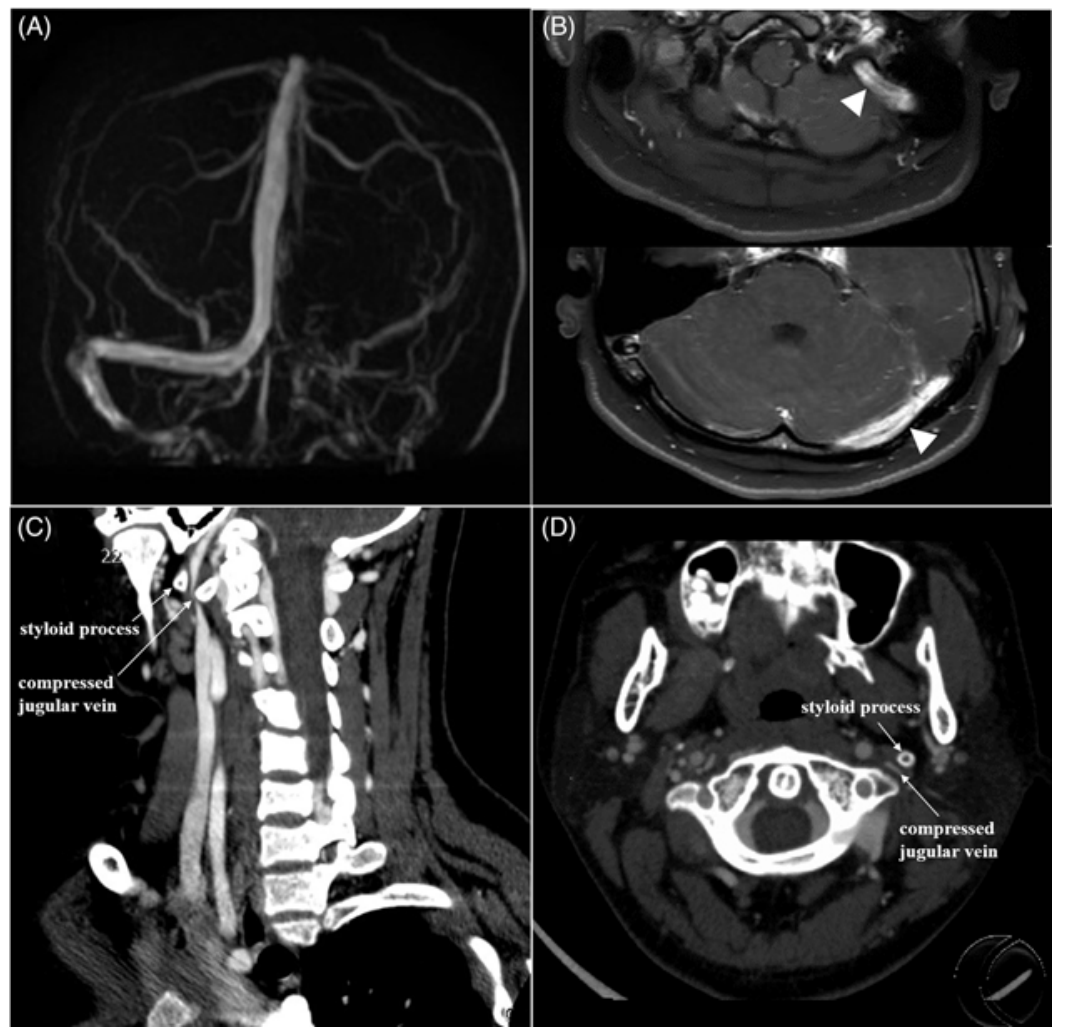

Figure 1: Neurological imaging examinations of the patient. PC-MRV (A) showed absent signal in the left transverse-sigmoid sinuses. Contrast-enhanced $3 D$ fat-saturated T1 VISTA MRI ( $B$; white arrowheads) demonstrated gadolinium enhancement of the left transverse-sigmoid sinuses, indicating chronic venous sinus thrombosis. The curved planar reformation $(C P R)$ and axial source images from $C T$ venography images $(C$ and $D$; white arrow) showed styloid process compressing the left jugular vein.

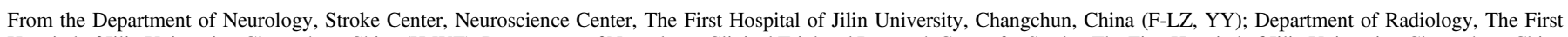

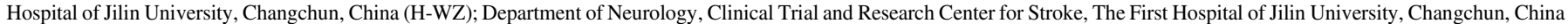
(Z-NG, YY)

Received October 16, 2018. Final Revisions Submitted February 1, 2019. Date of Acceptance February 5, 2019.

*These two authors contributed equally to the manuscript.

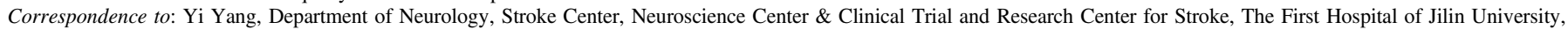
Xinmin Street 71\#, Changchun, Jilin 130021, China. Email: doctoryangyi@163.com; doctor_yangyi@hotmail.com 
A 15-year-old teenager presented with a 2-month history of headache. Neurological examination was normal except for papilledema. Further lumbar puncture indicated intracranial hypertension $\left(330 \mathrm{~mm} \mathrm{H}_{2} \mathrm{O}\right)$. Brain magnetic resonance imaging (MRI) was normal but phase contrast-magnetic resonance venography (PC-MRV) (Figure 1(A)) suggested possible left transverse-sigmoid sinus thrombosis; subsequent contrast-enhanced 3D fat-saturated $\mathrm{T} 1$ volumetric isotropic turbo spin echo acquisition (VISTA) MRI (Figure 1(B)) confirmed the pathology. Hyper-coagulable panel results (including six steroid sex hormones, antithrombin III, protein C, protein $\mathrm{S}$, lupus anticoagulant, and anticardiolipin antibodies) were all within normal range. In further examination, computed tomography (CT) venography images (Figure $1(\mathrm{C})$ and (D)) showed that the left jugular vein was compressed by the styloid process, consistent with Eagle syndrome. ${ }^{1}$ The patient who refused the recommended surgical treatment, however, chose anticoagulant therapy consisting of low-molecular weight heparin subcutaneous injection in addition to new oral anticoagulant. At 18-month follow-up, the patient reported no symptoms remained.

Eagle syndrome, also known as styloid syndrome, was initially described by Watt Weems Eagle in 1937; it often presented with cervical pain on the side of the elongated styloid process, foreign-body sensation in the throat, and dysphagia. ${ }^{2}$ In addition, it can cause transient ischemic attacks or stroke due to cervical internal carotid artery compression. ${ }^{1}$ However, cases of Eagle syndrome causing cerebral venous sinus thrombosis were seldom reported. ${ }^{3}$ In our report, we want to remind the neurologists that
Eagle syndrome should be taken into account when dealing with cases of cerebral venous sinus thrombosis of unknown origin, and $\mathrm{CT}$ venography may be a useful diagnostic tool.

\section{Statement of Authorship}

Study concept and design: Yang; acquisition of data: Zhang, Zhou, and Guo; image analysis: Zhou; drafting of the manuscript: Zhang, Zhou; critical revision of the manuscript for important intellectual content: Yang; obtained funding: Yang.

\section{ACKNOWLedgements}

This research was supported by the National Key R\&D Program of China (2016YFC1301600), JLUSTIRT (2017TD12), and Jilin the Fundamental Research Funds for the Central Universities to YY.

\section{Disclosures}

The authors declare no conflicts of interest.

\section{REFERENCES}

1. Badhey A, Jategaonkar A, Anglin Kovacs AJ, et al. Eagle syndrome: a comprehensive review. Clin Neurol Neurosurg. 2017;159: 34-8.

2. Eagle WW. Elongated styloid processes: report of two cases. Arch Otolaryngol. 1937;25(5):584-7.

3. Aydin E, Quliyev H, Cinar C, Bozkaya H, Oran I. Eagle syndrome presenting with neurological symptoms. Turk Neurosurg. 2018;28(2):219-25. 